\title{
Direct comparison of steroid and non-steroid eluting small surface pacing leads: Randomized, multicenter clinical trial
}

\author{
Valentina Kutyifa ${ }^{1}$, Endre Zima ${ }^{1}$, Levente Molnar ${ }^{1}$, Christian Kuehne ${ }^{2}$, \\ Soehnke Theiss ${ }^{3}$, Gundula Herrmann ${ }^{4}$, Laszlo Geller ${ }^{1}$, Bela Merkely ${ }^{1}$; \\ on behalf of SIELLO PILOT study Investigators \\ ${ }^{1}$ Semmelweis University, Heart Center, Budapest, Hungary \\ ${ }^{2}$ University Leipzig, Cardiology/Angiology, Leipzig, Germany \\ ${ }^{3}$ Johannes Wesling Hospital Minden, Cardiology, Minden, Germany \\ ${ }^{4}$ Biotronik SE \& Co. KG, Berlin, Germany
}

\begin{abstract}
Background: The aim of the study was to evaluate the efficacy and safety of small surface steroid-eluting atrial and ventricular pacing leads in comparison to non-steroid leads using remote monitoring system (Biotronik Home Monitoring ${ }^{\circledR}$ ).

Methods: In this randomized multicenter prospective trial, SIELLO T steroid-eluting ventricular leads $(n=42)$ were compared to BPPU T non-steroid leads $(n=46)$ and SIELLO $J T$ steroid-eluting atrial leads $(n=24)$ to BPPU JT non-steroid leads $(n=27)$ (Biotronik, Berlin, Germany) in pacemaker devices with remote monitoring capabilities. Lead parameters were evaluated during implantation, at 1-week and 1, 3, 6-month outpatient follow-up. Remote monitoring data were collected weekly.

Results: Atrial and ventricular steroid-eluting leads had stable sensing and impedance as compared to non-steroid leads at implantation and during follow-up. Patients with non-steroid atrial leads had significantly higher threshold compared to steroid leads at 1-week and at 1, 3, 6-month follow-up with a peak at 1-month (1-month $1.4 \pm 0.6 \mathrm{vs} .0 .7 \pm 0.3 \mathrm{~V}$ at $0.4 \mathrm{~ms}$, $p<0.001 ; 6$-month $0.3 \pm 0.5$ vs. $0.2 \pm 0.3 \mathrm{~V}$ at $0.4 \mathrm{~ms}, p=0.002)$. Patients with non-steroid ventricular leads had significantly higher threshold compared to steroid leads at 1, 3, 6-month (6-month $1.0 \pm 0.3$ vs. $0.6 \pm 0.2 \mathrm{~V}$ at $0.4 \mathrm{~ms}, p<0.001)$. Remote monitoring confirmed consistent results. During the study, 3 patients died of non-lead-related death. Lead repositioning was necessary in 2 atrial, 2 ventricular steroid leads and in 1 ventricular non-steroid lead.
\end{abstract}

Conclusions: Atrial and ventricular pacemaker leads with steroid showed significantly lower pacing threshold compared to non-steroid leads, confirmed by remote monitoring. (Cardiol J 2013; 20, 4: 431-438)

Key words: steroid-eluting pacing leads, atrial pacing lead, ventricular pacing lead, remote monitoring, steroid

Address for correspondence: Bela Merkely, MD, PhD, DSc, Semmelweis University, Heart Center, Varosmajor utca 68. Budapest, H-1122, Hungary, tel: +36-1-4586840, fax: +36-1-4586842, e-mail: merkely.bela@kardio.sote.hu

Received: 10.12.2012 Accepted: 10.01.2013 


\section{Introduction}

Rising pacing threshold after implantation is a well-known phenomenon in endocardial pacing, caused by formation of a fibrotic capsule at the lead-tissue interface due to inflammatory reactions [1]. Use of anti-inflammatory drugs like dexamethasone has been established for many years to suppress acute and chronic cellular inflammation and release of inflammatory mediators, resulting in lower pacing threshold and pacing energy $[1,2]$.

With the development of pacemaker (PM) therapy, rate responsive sensors and enhanced diagnostic features necessitated the improvement of lead technology to reduce current drain and extend PM longevity [3]. Small surface, high-impedance pacing leads were able to decrease current drain, however the smaller surface area was initially associated with worse sensing capabilities and higher pacing threshold. Several studies have proven the beneficial short and long-term effects of steroid pacing leads [4-7], however, no study has been conducted yet to directly compare small surface endocardial pacing leads with or without steroid-eluting collar with other with identical shape and structure.

Home Monitoring ${ }^{\circledR}$ is an established remote monitoring system providing remote patient follow-up and monitoring while using automatic wireless communication and home transmitters. It is a reliable method to effectively reduce hospital visits [8,9], hospital visit related costs and improve patient safety $[10,11]$. The use of remote monitoring system has not yet been established to follow-up acute and chronic lead performance in PM patients with steroid or non steroid-eluting leads.

This randomized, multicenter clinical study was designed to compare small porous surface atrial and ventricular pacing leads with same shape and structure, with $0.27 \mathrm{mg}$ of dexamethasone acetate (DXA) collar or with no steroid-eluting surface.

\section{Methods}

\section{Patient population}

Patients included in the analysis were consecutively selected from 3 investigational international centers ( 2 centers in Germany, 1 center in Hungary) based on pre-specified inclusion and exclusion criteria. Inclusion criteria included patients with standard PM indication referred to the hospital if they were geographically stable and available for follow-up visits at the center. Patients were excluded if they had life expectancy of less than 6 months, planned cardiac surgery in the next 6 months, enrolled in another cardiac clinical investigation or had other medical devices that may have had an interaction with the implanted PM. The clinical investigation was conducted according to the ethical principles of the Declaration of Helsinki (2008) and ISO 14155 Clinical Investigation of Medical Devices (Part I and II 2003). The study protocol was approved by the International Ethics Committee (Freiburger Ethik-Kommission International, FEKI) in Germany and by the local Institutional Review Board (IRB) in Hungary. All patients provided informed consent before the enrollment. From October 2009 to August 2010, 88 patients were implanted with study investigational leads and conventional PM devices capable of remote monitoring. Six drop-outs occurred during the duration of the study due to patient death or loss of contact.

\section{Investigational leads}

The SIELLO T/JT leads are a family of $5.9 \mathrm{~F}$, steroid-eluting, transvenous, endocardial bipolar passive-fixation leads. The SIELLO T is a straight lead, while the SIELLO JT carries a J-shaped distal end. The SIELLO T lead is available in $53 \mathrm{~cm}$ and $60 \mathrm{~cm}$ length, while the SIELLO JT lead varies in lengths of $45 \mathrm{~cm}$ and $53 \mathrm{~cm}$. The leads have an isodiametric structure and silicone insulation. The inner and outer conductors consist of quadruple wire coils. They are covered by polyurethane overlay for better gliding. The SIELLO T/JT leads have an IS-1 connector and a $12 \mathrm{~mm}$ pole distance. The SIELLO T/JT electrode tip has a fractal iridium coating and an active surface area of $2.1 \mathrm{~mm}^{2}$. The ring electrode has a surface of $17.4 \mathrm{~mm}^{2}$ and is coated with iridium. The leads carry 4 tines for passive fixation. The SIELLO T/JT leads have a dexamethasone eluting steroid collar with a total amount of $0.27 \mathrm{mg}$ DXA.

The BPPU T/JT leads are completely identical in shape and structure to the SIELLO T/JT leads but not featuring steroid eluting collar. Instead of the steroid eluting collar, there is a pure silicone dummy collar mounted with the same dimensions as the steroid eluting collar of the SIELLO T/JT leads.

\section{Randomization procedure}

Patients were randomized in a $1: 1$ fashion to receive either steroid-eluting SIELLO T/JT investigational lead or non-steroid BPPU T/JT investigational lead with the same shape and structure. 
Table 1. Indications of permanent pacemaker therapy.

\begin{tabular}{lccc}
\hline Indications & SIELLO T/JT (n= 42)* & BPPU (n= 46)* & Total (n = 88)* \\
\hline Intermittent or complete AVB & $18(42.9 \%)$ & $19(41.3 \%)$ & $37(42.0 \%)$ \\
Sinus node arrest, symptomatic bradycardia & $20(47.6 \%)$ & $16(34.8 \%)$ & $36(40.9 \%)$ \\
Sinoatrial block & $1(2.4 \%)$ & $2(4.3 \%)$ & $3(3.4 \%)$ \\
Chronotropic incompetence & $2(4.8 \%)$ & $2(4.3 \%)$ & $4(4.5 \%)$ \\
Atrial tachycardia & $7(16.7 \%)$ & $7(15.2 \%)$ & $14(15.9 \%)$ \\
Other (indication) & $12(28.6 \%)$ & $18(39.1 \%)$ & $30(34.1 \%)$ \\
\hline *Due to multiple selections the quantities do not sum to the total of patients; AVB - atrioventricular block; Other - indication was not \\
specified
\end{tabular}

After patient enrollment, a sealed randomization envelope was assigned to the patient. The study was not structured to be blinded, the randomization envelope was opened by the study personnel and both the implanting physician and patients were aware of the treatment assignment.

\section{Device and lead implantation procedure}

All patients had undergone conventional transvenous PM implantation based on current standards. Commercially available single and dual chamber PM devices (Biotronik) were used in the study with remote monitoring capabilities available. Lead and device testing during the implantation procedure was performed based on accepted standards. No additional test was required by the study protocol.

\section{Device programming}

Devices were programmed to AAI(R), $\mathrm{VVI}(\mathrm{R})$ or $\mathrm{DDD}(\mathrm{R})$ mode depending on the leads and PM implanted. Devices with Home Monitoring ${ }^{\circledR}$ available were recommended to program it $\mathrm{ON}$, unless not eligible for the patient.

\section{Patient follow-up, device interrogation}

Patients had an ambulatory follow-up within 1-week after device implantation (pre-discharge testing) and 1,3,6-month thereafter until the end of the trial or in case of an adverse event. The mean follow-up time of the study was 6 months. Home Monitoring ${ }^{\oplus}$ data were received and evaluated in patients with remote monitoring on a daily basis. Interrogation data at regular follow-up procedures and at any device-related adverse events were sent to the interrogation core laboratory in Berlin, Germany for further evaluation.

\section{Definitions and study endpoints}

The primary end point of the study was the efficacy assessment of SIELLO T/JT leads suppor- ted by the collection of measured data (sensing, pacing impedance, and pacing threshold) during the implantation and follow-up procedures with special focus on the atrial and ventricular threshold evaluated at $0.4 \mathrm{~ms}$ impulse width and compared to the non-steroid BPPU T/JT leads. The secondary endpoint was lead safety data on adverse events collected during the entire study duration.

\section{Statistical analysis}

Continuous variables are expressed as mean \pm standard deviation. Categorical data are summarized as frequencies and percentages. The randomization process was carried out using PROC PLAN in SAS with a block length of 6 . The lead evaluation data were compared between patient groups of steroid-eluting and non steroid-eluting pacing leads, T-test for independent samples or Mann-Whitney-U test was used, as appropriate. All tests were 2 -sided and a p-value of $<0.05$ was considered statistically significant.

\section{Results}

Steroid or non-steroid eluting leads were implanted in 88 patients ( 35 female) with the mean age of $72.9 \pm 10.1$ years in the steroid-eluting lead group ( 42 patients) and $73.4 \pm 9.1$ years in the non steroid-eluting lead group (46 patients).

\section{Clinical characteristics}

Patients with implanted non-steroid lead had trend towards more frequent valvular disease ( $30.4 \%$ vs. $16.7 \%, p=0.130$ ) and cardiomyopathy $(17.4 \%$ vs. $7.4 \%, \mathrm{p}=0.147)$. Incidence of hypertension, diabetes mellitus and renal insufficiency and drug treatment was similar in both groups (data not shown).

Indication of permanent PM therapy is summarized in Table 1 . Twenty (47.6\%) patients with steroid-eluting leads and 16 (34.8\%) patients with 
Table 2. Distribution of implanted leads per patient and implanted pacemaker models.

\begin{tabular}{lc}
\hline Leads & $27(30.7 \%)$ \\
BPPU JT and BPPU T & $24(27.3 \%)$ \\
SIELLO JT and SIELLO T & $15(17.0 \%)$ \\
SIELLO T and non-study & \\
RA lead & $14(15.9 \%)$ \\
BPPU T and non-study & \\
RA lead & $5(5.7 \%)$ \\
BPPU T only & $3(3.4 \%)$ \\
SIELLO T only & $88(100 \%)$ \\
Total & \\
Pacemaker model & $5(5.7 \%)$ \\
Philos II SR & $13(14.8 \%)$ \\
Philos II DR & $19(21.6 \%)$ \\
Philos II DR-T & $1(1.1 \%)$ \\
Cylos VR & $3(3.4 \%)$ \\
Cylos DR & $2(2.3 \%)$ \\
Cylos 990 SR & $13(14.8 \%)$ \\
Cylos 990 DR & $30(34.1 \%)$ \\
Cylos 990 DR-T & $2(2.3 \%)$ \\
Evia DR-T & $88(100 \%)$ \\
Total & \\
\hline
\end{tabular}

All pacemaker devices manufactured by Biotronik SE \& Co. KG, Berlin, Germany and have valid CE-certificate.

non-steroid leads had symptomatic sinus arrest at baseline. Intermittent or complete atrioventricular block was present in $18(42.9 \%)$ patients with steroid-eluting leads and $19(41.3 \%)$ patients with non-steroid leads.

\section{Lead and device implantation procedure}

Implantation of right atrial leads was primarily performed via left cephalic access $(60.9 \%)$, less often via the subclavian vein $(39.1 \%)$. Ventricular leads were implanted via the left or right cephalic vein $(57.8 \%)$, less often via the left or right subclavian vein $(42.2 \%)$.

The distribution of implanted leads per patient and implanted PM models are summarized in Table 2. Non-steroid BPPU JT atrial lead and BPPU T ventricular leads were implanted in $30.7 \%$ of the patients, while steroid-eluting SIELLO JT atrial lead and SIELLO T ventricular lead were present in $27.3 \%$ of the patients. Single-chamber devices and ventricular lead only with steroid-eluting tip were implanted in $3.4 \%$ of the patients, in $5.7 \%$ with no steroid tip. Both study and non-study leads were implanted in $15.9 \%$ and $17.0 \%$ of the total patient population, respectively.

The ventricular lead position was apical in $45.2 \%$ of steroid eluting leads and $43.5 \%$ of non- -steroid leads, while septal in $54.8 \%$ of steroid-eluting leads and $56.5 \%$ of non-steroid leads. The atrial lead was implanted at the right atrial appendage in all patients.

Lead implantation procedure as assessed by the implanting physician was very easy or easy in all atrial leads (100\%), while declared to be the same in $96.5 \%$ of ventricular leads. Moderately difficult or difficult implantation of the ventricular leads of either type was reported in $3.5 \%$ of the total patient population.

The total fluoroscopy time was $2.8 \pm 2.7 \mathrm{~min}$ (median: 1.8, minimum: 0.1, maximum: 8.0) in single atrial or ventricular lead implantations, while $4.1 \pm 3.4$ min (median: 3.0 , minimum: 0.3 , maximum: 18.5) in case of dual chamber devices. The procedure time was $26.4 \pm 8.3$ min (median: 22.5, minimum: 19.0, maximum: 40.0) for single chamber PM and 28.0 \pm 13.8 min (median: 27.0, minimum: 8.0, maximum: 75.0) for dual chamber PM.

\section{Evaluation of lead integrity and lead parameters, device longevity}

Atrial lead integrity parameters and sensing function evaluated at implantation, pre-discharge follow-up and at 1, 3, 6 months follow-up procedures were within normal limits in steroid and non-steroid eluting atrial leads. There was a significant difference found steroid-eluting and non steroid-eluting atrial leads in the pacing impedance at 1 -month follow-up (1-month $774 \pm 91$ vs. $700 \pm$ \pm 111 Ohm, $\mathrm{p}=0.012$ ).

The atrial threshold was significantly higher in non-steroid leads as compared to steroid-eluting leads at 1 -week and at 1, 3 and 6 month follow-up with a peak at 1 -month (1-month $1.4 \pm 0.6$ vs. $0.7 \pm 0.3 \mathrm{~V}$ at $0.4 \mathrm{~ms}, \mathrm{p}<0.001$ ) (Table 3 ). The mean value of atrial threshold in non-steroid leads was increasing until the 3-month follow-up, and slightly decreasing until the 6-month follow-up, then remained stable (Fig. 1).

Ventricular steroid-eluting leads had stable and similar sensing and lead impedance as compared to non-steroid leads at implantation and during follow-up. The ventricular pacing impedance was slightly higher in steroid-eluting leads at pre-discharge follow-up as compared to non-steroid-eluting ventricular leads $(911 \pm 138$ vs. $840 \pm$ $\pm 128 \mathrm{Ohm}, \mathrm{p}=0.015)$. Patients with non-steroid ventricular leads had significantly higher threshold compared to steroid-eluting leads at 1, 3, 6-month follow-up visits with clear early separation at 1 month, remaining stable at the 6 -month follow-up (6-month $1.0 \pm 0.3$ vs. $0.6 \pm 0.2 \mathrm{~V}$ at $0.4 \mathrm{~ms}$, $\mathrm{p}<0.001)$ (Table 3). 
Table 3. Atrial and ventricular pacing threshold of SIELLO JT and BPPU JT leads.

\begin{tabular}{|c|c|c|c|c|c|}
\hline Procedure & Lead type & $\mathbf{N}$ & Median & SD & $\mathbf{P}$ \\
\hline \multicolumn{6}{|c|}{ Atrial pacing threshold } \\
\hline Implant & $\begin{array}{l}\text { SIELLO JT } \\
\text { BPPU JT }\end{array}$ & $\begin{array}{l}21 \\
23\end{array}$ & $\begin{array}{l}0.6 \\
0.6\end{array}$ & $\begin{array}{l}0.3 \\
0.5\end{array}$ & 0.447 \\
\hline PHD & $\begin{array}{l}\text { SIELLO JT } \\
\text { BPPU JT }\end{array}$ & $\begin{array}{l}19 \\
24\end{array}$ & $\begin{array}{l}0.5 \\
0.6\end{array}$ & $\begin{array}{l}0.1 \\
0.6\end{array}$ & 0.039 \\
\hline 1 month FU & $\begin{array}{l}\text { SIELLO JT } \\
\text { BPPU JT }\end{array}$ & $\begin{array}{l}19 \\
24\end{array}$ & $\begin{array}{l}0.6 \\
1.5\end{array}$ & $\begin{array}{l}0.3 \\
0.6\end{array}$ & $<0.001$ \\
\hline 3 month FU & $\begin{array}{l}\text { SIELLO JT } \\
\text { BPPU JT }\end{array}$ & $\begin{array}{l}20 \\
25\end{array}$ & $\begin{array}{l}0.6 \\
0.8\end{array}$ & $\begin{array}{l}0.3 \\
0.3\end{array}$ & 0.047 \\
\hline 6 month FU & $\begin{array}{l}\text { SIELLO JT } \\
\text { BPPU JT }\end{array}$ & $\begin{array}{l}20 \\
22\end{array}$ & $\begin{array}{l}0.6 \\
0.8\end{array}$ & $\begin{array}{l}0.2 \\
0.3\end{array}$ & 0.002 \\
\hline \multicolumn{6}{|c|}{ Ventricular pacing threshold } \\
\hline Implant & $\begin{array}{l}\text { SIELLO T } \\
\text { BPPU T }\end{array}$ & $\begin{array}{l}39 \\
43\end{array}$ & $\begin{array}{l}0.4 \\
0.4\end{array}$ & $\begin{array}{l}0.2 \\
0.1\end{array}$ & 0.603 \\
\hline PHD & $\begin{array}{l}\text { SIELLO T } \\
\text { BPPU T }\end{array}$ & $\begin{array}{l}42 \\
45\end{array}$ & $\begin{array}{l}0.5 \\
0.5\end{array}$ & $\begin{array}{l}0.4 \\
0.2\end{array}$ & 0.377 \\
\hline 1 month FU & $\begin{array}{l}\text { SIELLO T } \\
\text { BPPU T }\end{array}$ & $\begin{array}{l}37 \\
43\end{array}$ & $\begin{array}{l}0.6 \\
1.0\end{array}$ & $\begin{array}{l}0.4 \\
0.4\end{array}$ & $<0.001$ \\
\hline 3 month FU & $\begin{array}{l}\text { SIELLO T } \\
\text { BPPU T }\end{array}$ & $\begin{array}{l}40 \\
43\end{array}$ & $\begin{array}{l}0.6 \\
0.9\end{array}$ & $\begin{array}{l}0.3 \\
0.2\end{array}$ & $<0.001$ \\
\hline 6 month FU & $\begin{array}{l}\text { SIELLO T } \\
\text { BPPU T }\end{array}$ & $\begin{array}{l}37 \\
38\end{array}$ & $\begin{array}{l}0.6 \\
1.0\end{array}$ & $\begin{array}{l}0.2 \\
0.3\end{array}$ & $<0.001$ \\
\hline
\end{tabular}

PHD — pre-discharge testing; FU — follow-up; SD — standard deviation

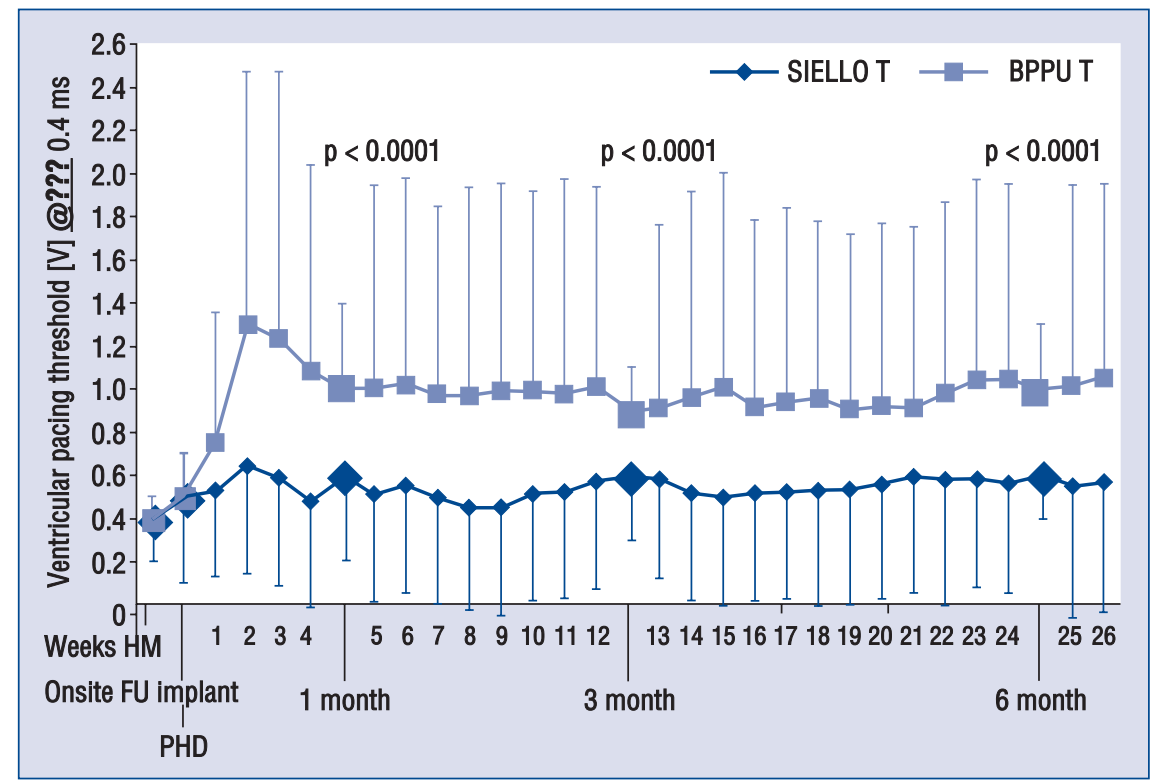

Figure 1. Ventricular pacing threshold values provided by Home Monitoring ${ }^{\circledR}(H M)$ and measured at on-site follow-up. The means of the onsite follow-up visit measurements are bold; PHD — pre-discharge testing; FU — follow-up.

Home Monitoring $^{\circledR}$ data of steroid-eluting and non-steroid ventricular lead performance were available in $51(58 \%)$ patients implanted with ventricular steroid or non-steroid leads. Figure 1 shows ventricular threshold data at on-site follow-up visits, as well as weekly Home Monitoring ${ }^{\circledR}$ data. We found excellent correlation between on-site follow-up visit assessments and Home Monitoring ${ }^{\circledR}$ data 
Table 4. Adverse events and serious adverse events.

\begin{tabular}{lccc}
\hline Category & Adverse event & Serious adverse event & All \\
\hline Patient death: cardiac & $0(0.0 \%)$ & $1(3.3 \%)$ & $1(2.2 \%)$ \\
Patient death: non-cardiac & $0(0.0 \%)$ & $2(6.7 \%)$ & $2(4.4 \%)$ \\
Right atrial lead related & $3(20.0 \%)$ & $2(6.7 \%)$ & $5(11.1 \%)$ \\
Right ventricular lead related & $2(13.3 \%)$ & $3(10.0 \%)$ & $5(11.1 \%)$ \\
Procedure related & $1(6.7 \%)$ & $1(3.3 \%)$ & $2(4.4 \%)$ \\
Medical & $4(26.7 \%)$ & $18(60.0 \%)$ & $22(48.9 \%)$ \\
Arrhythmias & $4(26.7 \%)$ & $3(10.0 \%)$ & $7(15.6 \%)$ \\
Pacemaker therapy related & $1(6.7 \%)$ & $0(0.0 \%)$ & $1(2.2 \%)$ \\
Total & 15 & 30 & 45 \\
\hline
\end{tabular}

provided (3-month correlation coefficient $=0.88$, $\mathrm{p}<0.001,6$-month $=0.89, \mathrm{p}<0.001$ ).

We also calculated the device longevity for the steroid vs. non-steroid-eluting ventricular leads, using the standard programming of VVI $60 \mathrm{bpm}$, $100 \%$ pacing, and a stimulation pulse width of $0.4 \mathrm{~ms}$. Patients implanted with a steroid-eluting lead had $2 \pm 5$ months additional device longevity, depending on the type of the implanted PM.

\section{Adverse events}

A total of 45 adverse events ( 0.51 events/ /patient) have been reported during the duration of the study. All adverse events are sorted by underlying cause and severity, as adverse event (AE) or serious adverse event (SAE) in Table 4. Two-thirds of AE's was classified as SAE. Seven AE's were reported as cases of atrial fibrillation. One AE was reported with symptoms of chronotropic incompetence, device reprogramming eliminated the symptoms. A total of 10 lead related adverse device effects occurred, 5 of them were resolved by PM reprogramming. Four SIELLO T/JT lead and $1 \mathrm{BPPU} \mathrm{T} / \mathrm{JT}$ lead required lead reposition procedure during the study, all of the lead dislodgements occurred in the early postoperative period (BPPU T non-steroid lead 1 day after implantation, SIELLO T/JT steroid leads $0-2$ days after implantation). During the follow-up 3 patients died, without any relation to the investigational study leads (1 of sepsis, 1 of multiple organ failure and 1 of sudden cardiac death).

\section{Discussion}

To our knowledge, this is the first randomized multicenter study directly comparing steroid-eluting and non-steroid eluting small surface atrial and ventricular lead performance. Our study demonstrated that atrial and ventricular small surface pacing leads with steroid collar had significantly lower pacing threshold compared to non-steroid leads. Both leads were safe and efficient as additionally confirmed by Home Monitoring ${ }^{\circledR}$.

Previous studies have shown that standard steroid-eluting pacing leads either with dexamethasone acetate or phosphate compound efficiently reduce the pacing threshold rise after implantation caused by inflammatory reactions at the tissue-lead interface $[1,7,12]$. This effect was stable and maintained during 5-or 10-year follow-up [13, 14]. Similarly beneficial effects of steroid-eluting coating were observed in active fixation atrial pacing leads [15].

Small surface, higher impedance pacing leads were able to reduce battery current drain, however the small surface area was associated with worse sensing capabilities and higher pacing threshold $[16,17]$. Therefore, there is a rationale to use steroid eluting surface in small surface pacing leads.

The main finding of the current study was that patients with non steroid-eluting atrial leads had significantly higher threshold compared to steroid-eluting leads at 1 -week and 1, 3 and 6-month follow-up. Consistent with these findings, patients with non-steroid ventricular leads showed significantly higher thresholds compared to steroid-eluting pacing leads at 1, 3, 6-month follow-up. Previous clinical studies showed similar results when comparing steroid-eluting conventional surface electrodes with non-steroid-eluting leads [18] or when comparing high-impedance steroid eluting leads with conventional surface steroid-eluting leads [5].

Additional strength of this trial is that remote Home Monitoring $^{\circledast}$ system was proven to be efficient, following changes in ventricular threshold 
parameters with an excellent correlation $(\mathrm{r}=0.89$ at 6 -month, $\mathrm{p}<0.001$ ). This might have important clinical implications in the future. Previous studies showed that Home Monitoring ${ }^{\circledR}$ effectively identified PM patients in need for in-office follow-up [8], reduced follow-up visits [8] and shortened the length of hospital stay at implantation [9]. It reduced hospitalizations caused by atrial arrhythmias and related strokes, improved patient safety and outcomes, while maintained quality of life [8]. Patients using the Home Monitoring ${ }^{\circledR}$ system were generally satisfied with the system [10]. The use of a remote monitoring system has not yet been established to follow-up acute and chronic lead performance in PM patients with steroid or non steroid-eluting leads. Patients with high pacing threshold at implantation might benefit from implantation of a PM with remote monitoring capabilities by reducing in-hospital stay and improving patient safety.

During the course of the trial four SIELLO T/ /JT lead and 1 BPPU T/JT leads had dislodgements, however all the dislodgements occurred in the early postoperative period, within the first 2 days after implantation. Given the time frame of the lead dislodgements, they are considered to occur due to the technical challenge of implanting this new type of electrodes, and not specifically attributed to the steroid coating.

The calculated device longevity indicated, that the implantation of a steroid-eluting lead is associated with a longer battery life, therefore replacement is less often needed, which has already been suggested earlier [15].

A potential limitation of our study might be the relatively small number of atrial leads implanted. Additionally, these leads were small surface leads, but the pacing impedance did not exceed $1000 \mathrm{Ohm}$. Remote monitoring was not available for atrial leads during the conduction of the current study however we assume that the excellent agreement with remote and on-site follow-up for ventricular leads might be similar for atrial leads. Longer-term follow-up is needed to further evaluate pacing threshold in patients with non-steroid leads, which might rise after 6 months.

\section{Conclusions}

This randomized, multicenter, large cohort study demonstrated that steroid-eluting atrial and ventricular small surface PM leads have significantly lower pacing threshold as compared to non steroid-eluting atrial and ventricular leads with the same shape and structure. Both leads were proven to be efficient and safe. Ventricular lead performance was additionally confirmed by remote monitoring (Home Monitoring ${ }^{\circledR}$ ).

Clinical Trial Registration: URL: http://clinicaltrials.gov. Unique identifier: NCT01000532.

Funding: The SIELLO PILOT study was supported by a research grant from Biotronik SE \& Co. KG, Berlin, Germany.

Conflict of interest: V.K. has received consultant fees/honoraria from Biotronik SE \& Co. KG and Servier, and research support from Boston Scientific. E.Z. has received consultant fees and honoraria from Boston Scientific, Innomed, Biotronik, Medtronic, and St. Jude Medical for lectures, training, and participation in clinical trials. G.H. is an employee of Biotronik SE \& Co. KG, Berlin, Germany. L.G. has received consultant fees/honoraria from Biotronik, Medtronic, St. Jude Medical, and Johnson \& Johnson. B.M. has received consultant fees/honoraria from Biotronik, Boston Scientific, Medtronic, and St. Jude Medical, and serves in the speaker's bureau of Boehringer Ingelheim. L.M., C.K., S.T. have no conflict of interest to disclose.

\section{References}

1. Radovsky AS, Van Vleet JF, Stokes KB, Tacker WA, Jr. Paired comparisons of steroid-eluting and nonsteroid endocardial pacemaker leads in dogs: Electrical performance and morphologic alterations. Pacing and clinical electrophysiology. PACE, 1988; 11: 1085-1094.

2. Radovsky AS, Van Vleet JF. Effects of dexamethasone elution on tissue reaction around stimulating electrodes of endocardial pacing leads in dogs. Am Heart J, 1989; 117: 1288-1298.

3. Yeh KH, Wang CC, Wen MS, Chou CC, Yeh SJ, Wu D. Long-term performance of transvenous, steroid-eluting, high impedance, passive-fixation ventricular pacing leads. Pacing and clinical electrophysiology. PACE, 2004; 27: 1399-1404.

4. Danilovic D, Breivik K, Hoff PI, Ohm OJ. Clinical performance of steroid-eluting pacing leads with $1.2-\mathrm{mm}^{2}$ electrodes. PACE, 1997; 20: 2799-2809.

5. Ellenbogen KA, Wood MA, Gilligan DM, Zmijewski M, Mans D. Steroid eluting high impedance pacing leads decrease short and long-term current drain: results from a multicenter clinical trial. CapSure Z investigators. PACE, 1999; 22: 39-48.

6. Moracchini PV, Cornacchia D, Bernasconi M et al. High impedance low energy pacing leads: Long-term results with a very small surface area steroid-eluting lead compared to three conventional electrodes. PACE, 1999; 22: 326-334.

7. Mond H, Stokes K, Helland J et al. The porous titanium steroid eluting electrode: A double blind study assessing the stimulation threshold effects of steroid. PACE, 1988; 11: 214-219. 


\section{Cardiology Journal 2013, Vol. 20, No. 4}

8. Mabo P, Victor F, Bazin P et al. A randomized trial of long-term remote monitoring of pacemaker recipients (The COMPAS trial). Eur Heart J, 2011; doi: 10.1093/eurheartj/ehr419.

9. Halimi F, Clementy J, Attuel P, Dessenne X, Amara W. Optimized post-operative surveillance of permanent pacemakers by home monitoring: The OEDIPE trial. Europace, 2008; 10: 1392-1399.

10. Ricci RP, Morichelli L, Santini M. Remote control of implanted devices through Home Monitoring technology improves detection and clinical management of atrial fibrillation. Europace, 2009; 11: 54-61.

11. Burri H, Senouf D. Remote monitoring and follow-up of pacemakers and implantable cardioverter defibrillators. Europace, 2009; 11: 701-709.

12. Wish M, Swartz J, Cohen A, Cohen R, Fletcher R. Steroid-tipped leads versus porous platinum permanent pacemaker leads: A controlled study. PACE, 1990; 13: 1887-1890.
13. Mond HG, Stokes KB. The steroid-eluting electrode: A 10-year experience. PACE, 1996; 19: 1016-1020.

14. Schwaab B, Frohlig G, Berg M, Schwerdt H, Schieffer H. Five-year follow-up of a bipolar steroid-eluting ventricular pacing lead. PACE, 1999; 22: 1226-1228.

15. Crossley GH, Brinker JA, Reynolds D et al. Steroid elution improves the stimulation threshold in an active-fixation atrial permanent pacing lead. A randomized, controlled study. Model 4068 Investigators. Circulation, 1995; 92: 2935-2939.

16. Joglar JA, Welch PJ, Wilkinson WE, Hamdan MH, Page RL. Initial experience with a high-impedance tined endocardial pacemaker lead: Evidence for increased lead failure. Am Heart J, 1997; 134: 161-164.

17. Kikuchi K, Abe H, Nagatomo T, Nakashima Y. Microdislodgment:A likely mechanism of pacing failure with high impedence small area electrodes. PACE, 2003; 26: 1541-1543.

18. Schuchert A, Kuck KH. Effects of local steroid elution on chronic pacing threshold and impedance of endocardial pacemaker leads. Cardiology, 1993; 83: 240-243. 